\title{
DISEÑO DE POLÍTICA Y CAPACIDADES ESTATALES: LAS RESPUESTAS DE BOGOTÁ PARA ATENDER LA EMERGENCIA EN SALUD POR COVID-19
}

\author{
Diana Paz-Gómez ${ }^{1}$ \\ dmpazfl@flacso.edu.ec \\ Facultad Latinoamericana de Ciencias Sociales, Ecuador \\ Sindy Pinzón Soler ${ }^{2}$ \\ spinzonsoler@gmail.com \\ Universidad de Castilla-La Mancha, España
}

\begin{abstract}
Este artículo analiza los problemas de coordinación entre el nivel nacional y local de gobierno, en la implementación de medidas de salud en Bogotá para atender la pandemia por Covid-19. Bajo un contexto de descentralización, en el que la Constitución Política de 1991 reconoce la autonomía territorial, pero en el que aún se mantiene una relación de dependencia con el gobierno central, la crisis sanitaria producto del aumento de casos positivos por Covid-19 deja en evidencia incoherencias sobre la toma de decisiones, vistas desde las capacidades de política del gobierno local. A partir de un análisis cualitativodescriptivo, se plantea que la interacción de las capacidades operacionales, organizativas y políticas, inciden en resultados efectivos de las medidas; mientras que la ausencia de algún tipo de capacidad, orienta las medidas a una falla política.
\end{abstract}

Palabras Claves: Covid-19, capacidades politicas, coordinación intergubernamental, negociación política, Bogotá.

Candidata a Doctora en Políticas Públicas-Flacso Ecuador. Maestra en Estudios urbanos, Flacso Ecuador. Politóloga, Universidad del Cauca-Colombia

2 Especialista en Gobernabilidad, Derechos Humanos y Cultura de Paz. Profesional en Relaciones económicas Internacionales, Universidad Autónoma de Colombia. 


\section{Policy design and STATE CAPACITIES: Bogota's RESPONSES TO THE HEALTH EMERGENCY BY COVID-19}

This article analyzes the problems of coordination between the national and local levels of government in the implementation of health measures in Bogotá to address the Covid-19 pandemic. In a context of decentralization, in which the Political Constitution of 1991 recognizes territorial autonomy, but in which a relationship of dependence with the central government is still maintained, the health crisis resulting from the increase of positive cases of Covid-19 reveals inconsistencies in decision making, as seen from the policy capabilities of the local government. Based on a qualitative-descriptive analysis, it is proposed that the interaction of operational, organizational and political capacities has an impact on the effective results of the measures, while the absence of some type of capacity leads to a political failure.

Key words: Covid-19, political capacities, intergovernmental coordination, political negotiation, Bogotá. 


\section{Introducción}

La enfermedad por Covid-19 se identificó por primera vez el 31 de diciembre del 2019 en Wuhan, provincia de Hubei en la República Popular de China. En principio, el nuevo tipo de neumonía, se asoció a trabajadores del mercado mayorista de mariscos en el sur de Wuhan (OMS, 2020a), a principios del 2020, el gobierno chino da a conocer la nueva cepa de coronavirus, relacionado con el Síndrome Respiratorio Agudo Grave SARS-CoV-2, conocido como Covid-19(OMS, 2020b). En los primeros días de enero del 2020, el virus se extiende a países como Tailandia y posteriormente a Europa y Norteamérica por vía aérea (OMS, 2020a). Para finales del mes, la OMS confirmó 7818 casos en todo el mundo, constituyéndose el brote como emergencia de salud pública de importancia internacional (ESPII),

Para el 26 de febrero del 2020, se confirma el primer caso en Brasil seguido de Ecuador, Argentina, Chile y Colombia (González et al., 2020). En los primeros noventa días de la presencia del virus en el país, Colombia presentó una tasa baja de contagio con 33.354 casos positivos a comparación de Brasil con 363.211, Chile con 99.688 o Perú con 178.914 casos positivos (Acosta, 2020) mientras tanto, Bogotá concentraba la mayoría de casos positivos, cifra que hasta el momento se mantiene constante. Para el 31 de marzo, contaba con 390 casos confirmados y 5 fallecidos, seguido por los departamentos de Antioquia, Cundinamarca, Huila, Risaralda y Valle, además de las ciudades de Barranquilla y Cartagena (OPS, 2020b).

Con la declaratoria de emergencia por parte de la OMS y aún sin registrar casos, el Ministerio de Salud y Protección social colombiano emitió la circular No. 005 del 11 de febrero de 2020 que brindaba a los entes territoriales directrices para prevenir, identificar y dar atención ante los posibles casos de Covid-19, al igual que planes de preparación y respuesta (Ministerio de Salud, 2020a). Esto permitió el despliegue de medidas por parte de las entidades administradoras de planes de beneficios en salud (EAPPB), las empresas prestadoras de servicios de salud público y privado (IPS) y gobiernos locales, enfocadas en la detección temprana, contención, atención y seguimiento epidemiológico.

La confirmación del primer caso por Covid-19 en Colombia llevó al gobierno central a emitir bajo resolución 385 del 12 de marzo la emergencia sanitaria a nivel nacional, adoptando medidas para mitigar y controlar la propagación y efectos del Covid-19, dada la condición del sistema de salud, que de acuerdo con la Dirección epidemiológica y Demografia del Ministerio de Salud, enfrentaba graves problemas para asumir una pandemia, seguido de la declaración del Estado de emergencia económica, social y ecológica, mediante Decreto 417 (Presidencia de la República, 2020). 
En principio, las estrategias gubernamentales se concentraron en fortalecer las capacidades operativas y organizativas de sus sistemas de salud, en lo referente a la demanda hospitalaria, educativa y de empleo, junto con las disposiciones nacionales y locales para mitigar el aumento de casos positivos. Un segundo momento estuvo marcado por estrategias para enfrentar el impacto económico negativo del primer pico de la pandemia, con la apertura gradual de establecimientos comerciales, medidas vigentes hasta la fecha, a pesar del segundo y tercer pico que obligó a tomar medidas de confinamiento y aislamiento en Bogotá.

Para responder a la pregunta de qué manera las capacidades de política del gobierno local inciden en el resultado de las medidas de salud para atender la pandemia por Covid-19 este artículo propone un análisis de tipo cualitativo-descriptivo retomando el marco de diseño de políticas (Howlett \& Mukherjee, 2018). Se plantea que la combinación entre capacidades políticas, operativas y organizativas y los instrumentos inciden en una coordinación entre los niveles de gobierno (central y local) que conlleva a resultados efectivos sobre la implementación de políticas; en tanto la falta de algún tipo de capacidad, explica los resultados no óptimos (o fallas políticas) de las medidas en salud.

Para ello, se exploran las capacidades políticas, operativas y organizacionales del gobierno local (Wu et al., 2018b) y se clasifican en tres dimensiones: fuerte, débil y no preparada (Capano et al., 2020). Los resultados de las medidas son identificados a partir del apoyo político y ciudadano al gobierno local (McConnell, 2016) versus el número de casos por contagio y muertes por cuenta del Covid-19. En este sentido, Bogotá se considera como un caso relevante para aplicar el marco analítico propuesto, dado el diseño institucional colombiano y distrital y los resultados finales de las medidas en salud.

La información utilizada incluye la revisión de informes institucionales, bases oficiales del Observatorio Covid-19 en América Latina y el Caribe de la Cepal, Secretaría de Salud de Bogotá, Ministerio de Salud de Colombia. Se recoge una revisión de noticias de medios oficiales y twitter. Los datos descriptivos en torno al Covid-19, son tomados de las bases de datos del Center for Systems Science and Engineering (CSSE) de Johns Hopkins University and Medicine y la Organización Mundial de la Salud. 


\section{El modelo de Capacidades de Política}

Las capacidades de política (policy capacity) se refieren a las competencias y capacidades necesarias en el proceso de la política pública (Wu et al., 2015). Son entendidas como el conjunto de habilidades y recursos de un gobierno para tomar decisiones inteligentes (Painter \& Pierre, 2005) y por tanto una condición previa necesaria para el logro de resultados óptimos (Bali \& Ramesh, 2018).

Los altos niveles de capacidad se vinculan a resultados superiores en las políticas públicas; mientras que los déficits de capacidad se asocian al fracaso o resultados no óptimos del proceso de políticas, limitan la coordinación del gobierno y evidencian diseños institucionales débiles (Peters \& Pierre, 2016). El modelo anidado propuesto por Wu et al., (2018a) comprende las competencias y capacidades en tres tipos: analítica, operativa y política y cada una en tres niveles, individual, organizativa y sistémica.

La capacidad analítica responde a los conocimientos técnicos del gobierno que permitan identificar herramientas adecuadas al momento de responder ante un problema de política (Mukherjee \& Bali, 2019). Esto implica que a nivel individual, el gobierno cuente con funcionarios con experiencia o asesores expertos (Newman et al., 2016) a nivel organizativo, se cuente con capacidad de procesar información y datos y a nivel sistémico, con la facilidad, rapidez y disponibilidad de estos datos. La capacidad operacional hace referencia a la habilidad de anticipación de los diseñadores. A nivel individual, se asocia al desempeño de funciones de gestión o liderazgo, a nivel organizativo a la arquitectura del gobierno y a nivel sistémico, a la coordinación entre gobierno y agencias.

Tabla 1

Dimensiones y niveles de capacidades de política

\begin{tabular}{|c|c|c|c|}
\hline Dimensión/nivel & $\begin{array}{l}\text { Capacidades } \\
\text { Individuales }\end{array}$ & $\begin{array}{l}\text { Capacidades } \\
\text { Organizacionales }\end{array}$ & $\begin{array}{l}\text { Capacidades del } \\
\text { Sistema }\end{array}$ \\
\hline $\begin{array}{l}\text { Competencias } \\
\text { analíticas }\end{array}$ & $\begin{array}{l}\text { 1.Capacidad } \\
\text { de análisis } \\
\text { de políticas: } \\
\text { Conocimiento } \\
\text { de la política } \\
\text { sustantiva y de las } \\
\text { técnicas analíticas } \\
\text { y habilidades de } \\
\text { comunicación }\end{array}$ & $\begin{array}{l}\text { 2.Capacidades } \\
\text { organizativas de } \\
\text { información: } \\
\text { Arquitectura de } \\
\text { la información } \\
\text { y servicios } \\
\text { electrónicos; } \\
\text { sistema } \\
\text { presupuestario y } \\
\text { gestión de recursos } \\
\text { humanos }\end{array}$ & $\begin{array}{l}\text { 3. Capacidad } \\
\text { del sistema de } \\
\text { conocimientos: } \\
\text { Instituciones y } \\
\text { oportunidades } \\
\text { para la generación, } \\
\text { movilización y } \\
\text { utilización de } \\
\text { conocimientos }\end{array}$ \\
\hline
\end{tabular}




\begin{tabular}{|l|l|l|l|}
\hline Dimensión/nivel & $\begin{array}{l}\text { Capacidades } \\
\text { Individuales }\end{array}$ & $\begin{array}{l}\text { Capacidades } \\
\text { Organizacionales }\end{array}$ & $\begin{array}{l}\text { Capacidades del } \\
\text { Sistema }\end{array}$ \\
\hline $\begin{array}{l}\text { Competencias } \\
\text { Operacionales }\end{array}$ & $\begin{array}{l}\text { 4.Capacidad de } \\
\text { conocimientos } \\
\text { especializados: } \\
\text { Liderazgo; gestión } \\
\text { estratégica; } \\
\text { negociación y } \\
\text { resolución de } \\
\text { conflictos }\end{array}$ & $\begin{array}{l}\text { 5. Capacidad } \\
\text { de recursos } \\
\text { administrativos: }\end{array}$ & $\begin{array}{l}\text { Financiación; } \\
\text { dotación de } \\
\text { personal; niveles } \\
\text { de coordinación } \\
\text { intrainstitucional de } \\
\text { responsabilidad y } \\
\text { accountability: Estado } \\
\text { de derecho; sistema } \\
\text { judicial transparente }\end{array}$ \\
\hline $\begin{array}{l}\text { Competencias } \\
\text { Políticas }\end{array}$ & $\begin{array}{l}\text { 7. Capacidad de } \\
\text { aumento político: } \\
\text { Comprensión de } \\
\text { las necesidades y } \\
\text { posiciones de los } \\
\text { diferentes interesados; } \\
\text { juicio de viabilidad } \\
\text { política }\end{array}$ & $\begin{array}{l}\text { 8. Capacidad } \\
\text { política } \\
\text { organizativa: } \\
\text { Apoyo a los } \\
\text { políticos parte } \\
\text { de la institución; } \\
\text { niveles de } \\
\text { confianza y } \\
\text { comunicación } \\
\text { entre } \\
\text { organizaciones }\end{array}$ & $\begin{array}{l}\text { 9. Capacidad del } \\
\text { sistema político- } \\
\text { económico: } \\
\text { Legitimidad y } \\
\text { confianza pública; } \\
\text { recursos fiscales } \\
\text { adecuados }\end{array}$ \\
\hline
\end{tabular}

Fuente: (Howlett E Ramesh, 2016: 4)

Finalmente, la capacidad política (political) depende de la naturaleza de los acuerdos institucionales (Capano, 2020). A nivel individual, se identifica por las aptitudes de los profesionales de política en comunicar, negociar y crear consenso, a nivel organizativo por los recursos políticos con los que cuente un gobierno, como legitimidad, buena relación con ministros o comunicación con los stakeholders, y a nivel sistémico por el mantenimiento del apoyo político (Mukherjee \& Bali, 2018; Wu et al., 2018a).

Las capacidades políticas implican también una coordinación e interacción entre niveles de gobierno y diferentes agencias, con el fin de evitar conflictos entre programas $\mathrm{u}$ organizaciones en la toma de decisiones (Peters, 2018). Una débil coordinación, en un marco descentralizado, por lo general obliga a centralizar el diseño de las políticas (Geert et al., 2010), lo que produce acciones costosas e ineficaces (Cabrero Mendoza, 2007: 5). Mientras que una coordinación horizontal y vertical, compuesta de procesos políticos y técnicos con actores del gobierno central, sectores sociales, económicos y no gubernamentales, generan políticas participativas y concertadas que inciden en un diseño con resultados óptimos.

Estas competencias y recursos resultan esenciales para asumir la pandemia por Covid-19 puesto que contribuyen a generar respuestas efectivas; no obstante, la 
presencia de solo una capacidad adecuada no garantiza un desempeño eficaz en el proceso de políticas (Woo, 2020) puesto que se necesita de capacidad del Estado, acuerdos institucionales y tendencia al juego político que impulsen respuestas efectivas y eviten los conflictos interinstitucionales (Capano, 2020).

\subsection{Fallas en la implementación de políticas}

El fracaso de las políticas públicas no es todo o nada (McConnell, 2016). El resultado de las políticas varía entre algún logro (mínimo o sustantivo) hasta fracasos menores a mayores. El éxito se obtiene en la medida en que los objetivos propuestos se alcanzaron sin ninguna crítica de importancia y el apoyo es casi universal; por su parte, una política fracasa cuando no se alcanzan los objetivos propuestos y por ende no se recibe un apoyo claro (McConnell, 2010). Para definir la falla en la política se verifican atributos existentes como el alcance de objetivos originales, el impacto hacia los destinatarios, el avance sobre la resolución del problema, la visibilidad, la intencionalidad y la evitabilidad (Howlett, 2012).

La teoría plantea tres niveles de falla. La falla del programa involucra el alcance de las metas programáticas originales, con el costo inicial y dentro del periodo concebido inicialmente. Usualmente se asocia a la imposibilidad de lograr los resultados esperados en torno a los grupos beneficiarios. La falla del proceso se define a partir de la incapacidad de preservar objetivos o instrumentos del gobierno y de construir una coalición sostenible, esto implica que la política no cuenta con aprobación suficiente al pasar por puntos de veto y actores, así cuente con eficiencia o eficacia en términos de entrega de bienes y servicios. Finalmente, la falla política se deriva de la incapacidad de facilitar la tarea de gobierno, un bajo apoyo electoral y una oposición sustancial crítica del gobierno.

\section{El contexto por Covid-19 en Colombia y Bogotá}

Tras la confirmación del primer caso positivo por Covid-19 en Colombia a principios del mes de marzo de 2020, de una paciente procedente de Milán, el Ministerio de Salud y Protección Social despliega el Plan de respuesta y contingencia ante el Coronavirus en coordinación con secretarios de salud municipales, Entidades Promotoras de Salud (EPS), Instituciones Prestadoras de Salud (IPS) y aseguradoras (Ministerio de Salud, 2020b).

La mayor parte de casos positivos se concentraron en Bogotá. Para el 31 de marzo del 2020, la ciudad confirmaba 390 casos, de los cuales 316 se registraban en atención en casa, 31 pacientes en hospital y 19 en UCI con 5 fallecidos, seguido por Valle del Cauca que presentaba 116 casos, con 4 fallecidos y Antioquia con 101 casos (OPS, 2020b). La expansión rápida del virus llevó al Ministerio de Salud y Protección Social a declarar la fase de mitigación, sobre el cual se establecen medidas de confinamiento de niños hasta el 20 de abril del 2020 y mayores de 70 
años hasta el 30 de mayo (Ministerio de Salud, 2020c). Las primeras respuestas al sistema de salud, se dieron con la Resolución 522 del 28 de marzo del 2020, en la que se establecen los requisitos de importación y fabricación de reactivos para el diagnóstico de la enfermedad y adquisición de equipos médicos y biomédicos.

Dos meses después de conocerse el primer caso, Colombia ya reportaba 2.877.746 contagiados en total y 74.215 muertos (Hopkins University, 2021; Ministerio de Salud, 2021). Mientras tanto Bogotá de acuerdo con el Programa de Prueba, Rastreo y Aislamiento Sostenible (PRASS), ha mantenido cifras por encima de la tasa de contagio nacional, registrando el primer pico de la pandemia entre julio y agosto del 2020, tras la apertura gradual de actividades en el mes de junio. Seguido de los picos de noviembre del 2020 y enero del 2021 que duplicaron el número de casos de la primera ola de contagio.

Para enero del 2021, Colombia registró 1.908 .413 casos positivos, mientras que Bogotá registró 556.405 casos confirmados, muy por debajo siguió Medellín con 170.159 casos. Esta tendencia se mantiene en el número de fallecidos, Colombia registró 48.631 muertes y Bogotá 11.243 muertes, en este caso seguido muy por debajo se registra Cali con 3.041 y luego Medellín con 2.936 muertes (OPS, 2021), Para abril del 2021, Bogotá tiene una ocupación de UCI del 93,8\%.

Esto ha significado que del total de reportados con Covid-19 en el país al 1 de mayo del 2021, el 27,6\% se concentran en Bogotá, según datos del Ministerio de Salud, ascendiendo a la fecha a 793.537 confirmados, seguido del departamento de Antioquia con 472.591, Atlántico con 238.552 y Valle del Cauca con 238.277 (Ministerio de Salud, 2021). En referencia al género, de acuerdo con datos ofrecidos por la Secretaría de Salud Distrital, la tasa de mortalidad en hombres es mayor (271,9x 100.000 habitantes) en comparación con las mujeres (140 x 100.000 habitantes). En términos globales, Bogotá ocupa el sexto lugar en número de casos por millón de habitantes, detrás de ciudades como Miami, Nueva York, Madrid, Londres y otras urbes latinoamericanas (Saludata, 2021).

Al territorializar el contagio por Covid-19 en Bogotá, los desafios del gobierno local se han enfocado en atender la emergencia por localidad. La ciudad cuenta con 20 localidades (división política) de las cuales el 55\% de los casos por Covid-19 se concentran en Suba (15,2\%), Kennedy (13,3\%), Engativá (11,2\%), Usaquén (7,8\%) y Bosa $(7,4 \%)$. Las localidades con menores tasas de contagios por Covid-19 son Sumapaz con 20 casos positivos, La Candelaria, 4.332. 
Fuera de Bogotá, 8.342, Mártires, 10.896 y Antonio Nariño, 10.664 (ver tabla 1).

Tabla 2

Número de casos confirmados por localidad

\begin{tabular}{|c|c|c|c|c|}
\hline & & Localidad & Número de casos & Total localidades \\
\hline \multirow{5}{*}{$\begin{array}{l}50 \% \text { de los } \\
\text { casos }\end{array}$} & 1 & Suba & 114.275 & \multirow{5}{*}{$\begin{array}{l}412.970 \text { de un } \\
\text { total de } 793.537\end{array}$} \\
\hline & 2 & Kennedy & 99.616 & \\
\hline & 3 & Engativá & 84.598 & \\
\hline & 4 & Usaquén & 58.789 & \\
\hline & 5 & Bosa & 55.692 & \\
\hline \multirow{5}{*}{$\begin{array}{l}\text { Localidades } \\
\text { con menos } \\
\text { casos }\end{array}$} & 6 & Antonio Nariño & 10.664 & \\
\hline & 7 & Mártires & 10.896 & \\
\hline & 8 & Fuera de Bogotá & 8.342 & \\
\hline & 9 & La Candelaria & 4.332 & \\
\hline & 10 & Sumapaz & 20 & \\
\hline
\end{tabular}

Fuente: tomado del Observatorio de Salud de Bogotá, Saludata a 01 de mayo del 2021

\section{El despliegue de las medidas de salud para atender la emergencia sanitaria en Bogotá}

El inicio de la pandemia puso en marcha la declaratoria del Estado de emergencia por parte del gobierno nacional. A través de los Decretos 417 y 418 del 2020, el presidente Iván Duque declara el estado de emergencia social, económica y ecológica, acompañado del manejo del orden público para prevenir y controlar el Covid-19 (Ministerio del Interior, 2020). Estas primeras medidas se ajustaron a un modelo top down de gestión central a la local, puesto que, en el marco de la descentralización, los alcaldes y gobernadores mantuvieron la responsabilidad sobre la aplicación inmediata de las disposiciones en coordinación con el Ministerio de Salud, Secretarías de Salud y gobierno y Policía Metropolitana.

Los lineamientos del nivel nacional continuaron con la declaratoria de un nuevo estado de emergencia social, económica y ecológica por medio del Decreto 637 del 6 de mayo del 2020, con una duración de treinta días. Entre estas dos declaratorias se expidieron 63 y 42 decretos, respectivamente (Mendieta \& Tobón, 2020). Para el 25 de agosto el Decreto 1168 adopta el aislamiento selectivo y distanciamiento individual responsable en el marco de una reactivación económica y social en los municipios de alta afectación que dispone a los alcaldes a restringir actividades, áreas y zonas cuando consideren pertinentes, previo concepto y aprobación por parte del Ministerio de Salud. 
Sobre este escenario, los lineamientos distritales han considerado la promulgación de Decretos, Resoluciones y Circulares, vinculados algunos con Decretos Nacionales con incidencia local.Tras la confirmación del primer caso por Covid-19 en la ciudad, la Alcaldía de Bogotá expide la circular 024 del 2020 dirigida a las instituciones distritales para adoptar el teletrabajo y restricciones a comisiones al exterior (Secretaría General, 2020a). Mientras que la circular 026 del 2020 delimita los horarios de atención por parte de las instituciones públicas.

La primera respuesta del gobierno local, antes de la expedición del Decreto de estado de emergencia por parte del gobierno nacional, se concentró en el Decreto 081 de 2020, que adopta medidas sanitarias y acciones transitorias de policía para la mitigación de la situación de emergencia en Salud por cuenta del Covid-19 en concordancia con la Ley 1801 del 2016 que otorga a los gobiernos locales competencia extraordinaria de policía para atender situaciones de calamidad y la Ley 1523 del 2012 que otorga responsabilidad a los gobiernos subnacionales sobre la atención y gestión de riesgo. Esta medida estuvo acompañada de la adopción del Plan territorial de respuesta y la instalación del Consejo Distrital de Gestión de Riesgo y Cambio Climático de forma permanente (Secretaría General, 2020b).

Posterior a la declaratoria del estado de emergencia, el gobierno local expide la Resolución 712 del 2020, con lineamientos que establecen zonas de cuidado especial sobre las localidades con mayor número de contagios. Esto conllevó a formular planes diferenciados de contención al Covid-19 por localidad (Chapinero, Suba, Rafael Uribe, Bosa y Puente Aranda) sobre los cuales se implementan medidas de vigilancia epidemiológica, atención a grupos de riesgo, distanciamiento físico, uso estricto del tapabocas y realización de pruebas diagnósticas (Secretaría de Salud, 2020).

Con relación a la cooperación entre el gobierno local y departamental, los Decretos 090 del 19 de marzo y 091 del 22 de marzo del 2020, marcaron la implementación del Plan de aislamiento selectivo con el Plan Simulacro Vital, que llevó después al despliegue de estrategias de mitigación y contención del virus tras la expedición del Decreto Nacional 124 cuyo fin fue la reactivación económica sobre sectores estratégicos como manufactura, construcción, comercio al por menor y servicios no esenciales. A partir de una revisión documental, se identifican las principales medidas en salud implementadas por el gobierno de Bogotá, asociadas en tres dimensiones: distanciamiento social, sanitarias y atención al paciente. 
Tabla 3

Principales medidas de Salud implementadas en Bogotá

\begin{tabular}{|c|c|}
\hline Dimensiones & Medidas \\
\hline $\begin{array}{l}\text { Distanciamiento } \\
\text { social }\end{array}$ & $\begin{array}{l}\text { 1. Simulacro Vital desde el } 19 \text { de marzo del } 2020 \text { a las } 11: 59 \text { PM hasta } \\
\text { el } 24 \text { de marzo a las } 23: 59 \text { horas. } \\
\text { 2. Aislamiento preventivo obligatorio a partir del } 25 \text { de marzo del } \\
2020 \text { a las 00:00 horas hasta finales del mes de agosto tras declara- } \\
\text { toria de estado de emergencia. } \\
\text { 3. Limitación a la libre circulación de personas y vehículos con ex- } \\
\text { cepciones a la prestación en salud, adquisición de bienes de pri- } \\
\text { mera necesidad, desplazamiento a servicios bancarios, asistencia } \\
\text { y cuidado de niños y adultos mayores, actividades diplomáticas, } \\
\text { militares y de seguridad. } \\
\text { 4ico y género como primera medida de limitación a la circulación } \\
\text { y modificada por el Pico y cédula para circulación sobre el espacio } \\
\text { público con el último digito de la cédula de identificación } \\
\text { 5. Circulación de una sola persona por núcleo familiar para la adqui- } \\
\text { sición de bienes y servicios. } \\
\text { 6. Medida de distanciamiento individual responsable y teletrabajo } \\
\text { 7. Toque de queda nocturno, confinamiento social, confinamiento } \\
\text { estricto por localidades. } \\
\text { 8. Esquema } 4 x 3 \text { (4 días de actividad normal y } 3 \text { en cuarentena). } \\
\text { 9. Ley seca. } \\
\text { 10. Lavado de manos cada } 3 \text { horas, uso obligatorio de tapabocas y } \\
\text { distanciamiento social. }\end{array}$ \\
\hline Sanitarias & $\begin{array}{l}\text { 1. Atención a salud domiciliaria, virtual } \\
\text { 2. Priorización a grupos de mayor riesgo (mayores de } 70 \text { años y per- } \\
\text { sonas con comorbilidades) } \\
\text { 3. Pruebas de coronavirus gratis en diferentes localidades de Bogotá } \\
\text { 4. Distanciamiento social, teletrabajo, lavado de manos } \\
\text { 5. Estrategia DAR (Detecto, Aíslo y Reporto) dirigido desde las IPS }\end{array}$ \\
\hline $\begin{array}{l}\text { Atención al } \\
\text { paciente }\end{array}$ & $\begin{array}{l}\text { 1. Transferencias monetarias a hogares en pobreza o extrema pobreza } \\
\text { de acuerdo con categoría Sisbén. } \\
\text { 2. Canales de atención a la violencia de género en hogares. } \\
\text { 3. Distribución de renta básica girada por estrato socioeconómico } \\
\text { y localidad. } \\
\text { 4. Giros realizados para pago de arriendo solidario. } \\
\text { 5. Ayudas alimentarias a hogares en pobreza o pobreza extrema. } \\
\text { 6. Donatón Bogotá Solidaria en Casa para recolectar dinero y re- } \\
\text { cursos en especie para población en pobreza o extrema pobreza. } \\
\text { 7. Donación de plasma para ayudar a pacientes con Covid-19. } \\
\text { 8. Subsidios a servicios públicos. } \\
\text { 9. Jornadas de desinfección de estaciones y portales de Transmilenio. } \\
\text { 10. Seguimiento a personas contagiadas con Covid-19. }\end{array}$ \\
\hline
\end{tabular}

Fuente: Sobre la base del Observatorio de Salud de Bogotá (Saludata, 2021), Observatorio Cepal (CEPAL, 2020) y (Ministerio de Salud, 2021) 
Para el 2021, el Decreto 135 de abril 5 establece nuevamente medidas para atender el tercer pico de la pandemia, en torno a la restricción a la movilidad, expendio de bebidas alcohólicas y fortalecimiento del programa PRASS (Alcaldía de Bogotá, 2021). El Decreto 148 del 20 de abril, adopta medidas restrictivas a la movilidad en horas de la noche, pico y cédula, suspensión de procedimientos de baja, mediana o alta complejidad, continuación de trabajo en casa en instituciones del sector público y privado y fortalecimiento del programa PRASS, esto tras el aumento de ocupación de camas UCI (Alcaldía de Bogotá, 2021). Los demás decretos expedidos por el gobierno local, se han dirigido a la aplicación de lineamientos nacionales por parte del Ministerio de Salud.

\section{Capacidades del gobierno de Bogotá para atender la emergencia en Salud}

El marco de diseño de políticas propone un análisis de cohorte cualitativo sobre los resultados de las políticas públicas, es por ello que el modelo anidado propuesto por Wu et al (2015) ofrece una exploración hacia la caracterización de las capacidades con las que cuenta un gobierno, sea nacional o local, y lo vincula con un tipo de falla o efectividad dado en el diseño de las políticas públicas. Los resultados entre la vinculación de uno y otro elemento, hacen posible considerar el punto de quiebre de la toma de decisiones.

\subsection{Capacidades operativas-organizativas}

Las medidas implementadas por el gobierno de Bogotá se orientaron al distanciamiento social, sanitarias y de atención al paciente que obligaron al despliegue de capacidades operativas, analíticas y políticas para el cumplimiento de los objetivos. Al principio de la pandemia Bogotá contaba con 967 camas UCI al 22 de marzo del 2020, para el 1 de mayo del 2021 su capacidad aumentó a 2.466 camas UCI de las cuales 2.026 se destinaron a pacientes con Covid-19; no obstante, a pesar de este aumento, Bogotá presenta 1,8 camas UCI por cada 1.000 habitantes en una población que supera los siete millones (Saludata, 2021).

En este despliegue, la coordinación entre secretarías internas del distrito permitió la implementación de apoyos alimentarios a población en pobreza y extrema pobreza. Frente a la atención médica, la Resolución 521 de 2020 priorizó la atención telefónica, virtual y domiciliaria a población mayor o con factores de riesgo, lo que llevó al despliegue de 84 equipos domiciliarios, direccionados por el Centro Regulador de Urgencias y Emergencias CRUE (Rodríguez-Cuenca et al., 2020). Otra de las capacidades frente a la atención médica se orientó al acondicionamiento de camas de baja complejidad en Corferias, en coordinación con la Red hospitalaria de Bogotá y la Cámara de Comercio de Bogotá, en el que se habilitaron 2.000 cubículos para atención entre el mes de marzo y septiembre del 2020. En los 
primeros meses, además, el distrito ya contaba con la compra de 200.000 pruebas diagnósticas, con un objetivo de aplicación entre 1.400 y 3.000 pruebas diarias.

Frente a las medidas sanitarias y la implementación de la estrategia DAR (Detecto, Aíslo, Reporto) el gobierno local contó con la Entidad de Gestión Administrativa y Técnica (EGAT) para monitorear el mercado de medicamentos y mediante el mecanismo de red pública se realizó una compra unificada de medicamentos para abastecer centros médicos. En coordinación con actores privados e instituciones de salud como la Asociación Colombiana de Medicina Crítica y Cuidado Intensivo (AMCI) y la Sociedad Colombiana de Anestesiología y Reanimación (SCARE), se generaron lineamientos técnicos para atender a personas con Covid-19 (Alcaldía de Bogotá, 2020).

En cuanto a la comunicación, la estrategia de Bogotá ciudadana como sistema de información, permitió el seguimiento a personas contagiadas. El Observatorio de Salud de Bogotá diariamente ofrece información actualizada sobre el número de contagios y ocupación de camas UCI, en el que los ciudadanos tienen la oportunidad de solicitar información. Por su parte, en torno a la medida de toma de pruebas para detectar el Covid-19, la capacidad desplegada se orienta a la implementación de la página web saludcapital.com en donde las personas consultan sus resultados. Bogotá desarrolló también el modelo de medición de la curva del Covid-19 entre la Secretaría de Salud y expertos epidemiológicos, eso permite que mediante la página web los ciudadanos pueden acceder a las capacidades en camas UCI, las fuentes de contagio y puntos de atención.

La atención al paciente incluyó el fortalecimiento de capacidades del personal de salud de primera línea, priorizados también en la fase 1 de vacunación. En coordinación con la Agencia de Cooperación Internacional de Corea (KOICA) y la Organización Panamericana de la Salud (OPS), las estrategias se enfocaron en los hospitales públicos de Bogotá en los primeros seis meses de pandemia, con el propósito de desarrollar habilidades del personal de salud en primeros auxilios y detección temprana de síntomas de trastornos de la salud mental (OPS, 2020a). 
Tabla 4

Capacidades operativas y analíticas en Bogotá

\begin{tabular}{|c|c|c|c|}
\hline & Capacidad Individual & Capacidad Organizacional & Capacidad del Sistema \\
\hline 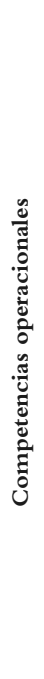 & $\begin{array}{l}\text {-Reconexiones al servicio público } \\
\text { de acueducto durante los prime- } \\
\text { ros meses de la pandemia: } 38147 \\
\text { reconexiones } \\
\text {-Reconexiones de servicio de gas } \\
\text { natural } \\
\text {-Montaje operacional para el plan } \\
\text { de vacunación } \\
\text {-Espacios destinados a pruebas de } \\
\text { identificación del Covid-19 de } \\
\text { forma gratuita para los ciudadanos } \\
\text {-Compra de Kits de bioseguridad } \\
\text { para dotar a comerciantes de las } \\
\text { localidades de Usme, Ciudad } \\
\text { Bolívar y Tunjuelito } \\
\text {-Adecuación del coliseo Movistar } \\
\text { Arena para vacunación masiva a } \\
\text { mayores de } 80 \text { años } \\
\text {-Adecuación de } 400 \text { puntos habi- } \\
\text { litados para vacunación masiva en } \\
\text { distintas fases }\end{array}$ & $\begin{array}{l}\text {-Adquisición de termocicladores } \\
\text { para laboratorios de salud públi- } \\
\text { ca, enfocados a la reducción de } \\
\text { tiempos de análisis de muestras } \\
\text {-Conformación de Red de labo- } \\
\text { ratorios para atender la demanda } \\
\text { de pruebas por Covid-19 } \\
\text {-Aumento de camas UCI } \\
\text { pasando de } 967 \text { camas en marzo } \\
\text { del } 2020 \text { a } 2.075 \text { para mayo } \\
\text { del } 2021 \\
\text { - Aplicación de Plan de vacuna- } \\
\text { ción en un millón de dosis al } 2 \\
\text { de mayo del } 2021\end{array}$ & $\begin{array}{l}\text {-Centro hospitalario de } \\
\text { Corferias con camas de baja } \\
\text { complejidad con } 274 \text { camas } \\
\text { hasta septiembre del } 2020 \\
\text {-Alianza entre Entidades } \\
\text { Promotoras de Salud, EPS del } \\
\text { régimen contributivo y subsi- } \\
\text { diado, IPS públicas y privadas } \\
\text { para implementar el plan de } \\
\text { expansión en salud }\end{array}$ \\
\hline 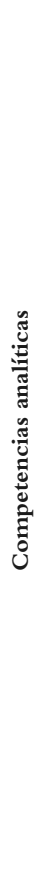 & $\begin{array}{l}\text {-Llamado al talento humano en } \\
\text { salud en ejercicio o formación, } \\
\text { para recibir entrenamiento de las } \\
\text { actividades a realizar. } \\
\text {-Reconocimiento económico } \\
\text { temporal para talento humano } \\
\text { de salud. } \\
\text { - Apoyo de expertos epidemio- } \\
\text { lógicos. } \\
\text {-Dirección Distrital de Relacio- } \\
\text { nes Internacionales (DDRI) para } \\
\text { compartir experiencias con el } \\
\text { mundo sobre la implementación } \\
\text { de medidas. }\end{array}$ & $\begin{array}{l}\text {-Contratación fija e incorpora- } \\
\text { ción a plana de personal médico } \\
\text { entre médicos, enfermeros y } \\
\text { auxiliares de enfermería. } \\
\text {-Plataforma virtual Bogotá Salud } \\
\text { Digital para agendamiento de } \\
\text { citas médicas, historia clínica y } \\
\text { entrega de medicamentos. } \\
\text {-SaluData: observatorio sobre } \\
\text { datos abiertos del Covid-19 en } \\
\text { Bogotá. } \\
\text { - Plataformas digitales con están- } \\
\text { dares básicos de audio y video } \\
\text { para el seguimiento de pacientes } \\
\text { con Covid-19. } \\
\text {-Página "Mi seguridad Social" } \\
\text { destinado a reportes de nove- } \\
\text { dades y consultas en el Sistema } \\
\text { General de Seguridad Social } \\
\text { en Salud y el Sistema General } \\
\text { de Riesgos Laborales a nivel } \\
\text { nacional. } \\
\text {-Donación de } 40 \text { ventiladores } \\
\text { y } 289 \text { equipos biomédicos para } \\
\text { las UCI. } \\
\text {-Sala blanca (producción far- } \\
\text { macéutica) para el desarrollo de } \\
\text { tratamientos celulares. }\end{array}$ & $\begin{array}{l}\text {-Centro Regulador de Urgen- } \\
\text { cias y Emergencias (CRUE) } \\
\text { para atender llamadas de } \\
\text { urgencia. } \\
\text {-Kiosco digital que permite } \\
\text { hacer uso de la plataforma } \\
\text { Bogotá Salud Digital en } \\
\text { localidades. } \\
\text {-Comité Epidemiológico } \\
\text { Nacional. } \\
\text {-Alianza EPS y CRUE para la } \\
\text { atención médica domiciliaria } \\
\text {-Servicio de atención prehos- } \\
\text { pitalaria de emergencias de } \\
\text { salud mental y apoyo psicoló- } \\
\text { gico con } 10 \text { equipos móviles } \\
\text { para prestar el servicio. } \\
\text {-Intervenciones de prevención } \\
\text { de conducta suicida y vio- } \\
\text { lencia de niños con el equipo } \\
\text { de Salud. } \\
\text {-Línea } 106 \text { para intervencio- } \\
\text { nes de casos de violencia. } \\
\text { - Equipo multidisciplinar para } \\
\text { campañas de prevención. }\end{array}$ \\
\hline
\end{tabular}

Fuente: Elaboración sobre la base de SaluData (2021) Alcaldía de Bogotá (2021) 


\section{$4.2($ In) capacidades políticas}

Las capacidades políticas implican coordinación. A su vez, están vinculadas con las fallas políticas que consideran el alto o bajo apoyo político a un gobierno local. Para el caso de Bogotá, Claudia López presenta una aprobación del 70\% y 71\% en los meses de agosto y octubre del 2020, respectivamente. A pesar de una baja porcentual de aprobación ciudadana, para el mes de abril del 2021 las encuestas arrojaron una aprobación del 52\% frente a una desaprobación del 44\%, que comenzó a subir en el mes de mayo con un $61 \%$ de aprobación, según encuesta realizada por INVAMER.

A pesar del apoyo político, el aumento en la desaprobación está asociada con los picos de la pandemia registrados tanto a nivel nacional como distrital. En tanto, frente a los casos confirmados por Covid-19, Bogotá mantiene las mayores cifras de contagio en comparación incluso con departamentos del país con un número de población similar. En términos de tasas de contagio por total de población, Colombia llega a un $7 \%$ con una población cercana a los 50 millones, frente a un 14\% de Bogotá con una población de 7 millones, por debajo está el departamento de Antioquia con una población de 6 millones, en que la tasa de contagio está alrededor del 8,6\% y para elValle del Cauca una tasa del 6,1\% (considerando que estos departamentos son los que presentan mayores tasas de contagio a nivel nacional).

En cuanto a las capacidades políticas, la interacción entre entidades locales se define como fuerte, si se combina con una organización y operación capaz de enfrentar la pandemia por Covid-19.

En el primer trimestre de la pandemia, se coordinaron acciones con actores privados para realizar la Donatón de recursos y alimentos destinados a la atención de población en pobreza y pobreza extrema. Otra de las acciones que llevaron a la cooperación fue la dada con Ecopetrol (entidad pública de carácter nacional) y la República de Corea para el desarrollo de estrategias de fortalecimiento al sector salud. En el afán de ampliar la capacidad de vacunación, el gobierno local comenzó diálogos para llegar a acuerdos en la producción de las vacunas AstraZeneca, Sinovac, Sputnik V y Jannsen.

Frente a la coordinación con actores privados, el foco del gobierno local ha estado enfocado en la cooperación con las EPS y clínicas del sector privado, a ello a través del programa "Unidos para seguir cuidándote", esto ha permitido poner en desarrollo medidas de seguimiento y control de pacientes con comorbilidades, madres lactantes y en embarazo. Si bien uno de los mayores conflictos presentados ha estado en las relaciones con el sector comercio, las capacidades en cuanto a participación de actores sociales y ciudadanía en general como taxistas, recicladores, tenderos, mensajeros, domiciliarios y lideres comunitarios, ha permitido la promoción de estrategias comunitarias como la implementación del TIPS (Territorios de Innovación y participación en Salud). 
La capacidad política a nivel de sistema refiere a las habilidades en cuanto a la cooperación entre distintos niveles de gobierno. En este caso, para Bogotá se clasifica una capacidad de débil a no presente. El marcado liderazgo local en el manejo de la pandemia comienza con la implementación del Simulacro Vital en coordinación con la Gobernación de Cundinamarca; sobre este hecho, la ciudadanía se pronunció por la falta de medidas por parte del gobierno central para el resto del territorio nacional, lo que incidió en la actuación por parte del gobierno para decretar el aislamiento obligatorio.

No obstante, las fallas en la coordinación entre niveles de gobierno se hicieron más visible tras la implementación del Decreto 438 de 2020 que señala la competencia central sobre las decisiones. La mandataria local, entonces, se opuso abiertamente a las medidas y/o anuncios por parte del presidente. Los canales de comunicación y difusión entre los mandatarios tomaron mayor fuerza en las redes sociales, provocando un escenario de incertidumbre entre la población tras las medidas encontradas que planteaban los dos gobiernos. El caso más significativo se dio en la apertura gradual del comercio y servicios en Bogotá y el cierre del aeropuerto El Dorado.

En este contexto, el gobierno nacional manifestó que los sectores de construcción y manufactura darían inicio al trabajo presencial escalado en la última semana de abril del 2020 como parte del plan de reactivación económica. Ante este anuncio la alcaldesa de Bogotá, tras la consulta con expertos epidemiológicos, argumentó que la medida podría provocar un incremento exponencial de casos positivos, puesto que esto implicaba un aumento en el uso del transporte público y la ciudad aún no estaba preparada para atender la movilidad de personas en el territorio (Semana, 2020).

Si bien el estado de emergencia implica medidas centralizadas, el gobierno local presentaba críticas en torno a la falta de discusión previa con los alcaldes para definir la proporcionalidad y efectividad de las medidas, de acuerdo a las condiciones de cada ciudad (El Tiempo, 2020). Ante este clima frío, la comunicación de los mandatarios se trasladó a twitter. En los primeros días, Claudia López pidió cerrar el aeropuerto El Dorado, con el fin de evitar el ingreso de personas contagiadas y así evitar el congestionamiento de camas UCI en hospitales, ello llevó al famoso tuit de la mandataria "sobre mi cadáver abren el aeropuerto el 27 de abril" (Twitter Claudia López), "esa sí sería una irresponsabilidad”, señaló en un programa radial, en esta misma declaración pidió el cierre de la frontera con Venezuela: "Con todo respeto me permito sugerir a las autoridades nacionales considerar el cierre de vuelos con Europa y otros países que ya tienen libre circulación de coronavirus Covid-19" (Twitter Claudia López).

La presión local también llevó a la renuncia del gerente del aeropuerto, tras no aplicar las medidas suficientes para contener el ingreso de personas contagiadas. Por su parte, el gobierno manifiesta que el cierre generaría un golpe a la economía colombiana, tanto en la entrada de turistas como grandes pérdidas de dinero de las 
aerolíneas, las empresas que prestan servicios a los viajeros y trabajadores informales alrededor del turismo.

Otro de los temas de conflicto entre los dos niveles de gobierno se generó en septiembre del 2020, tras las manifestaciones y disturbios que llevaron a miles de bogotanos a salir a las calles. Ante esta situación, la Alcaldía de Bogotá realiza un homenaje a las víctimas de presuntos abusos policiales que dejaron 14 muertos en Colombia, aunque asistió el Alto Comisionado para la Paz, el evento dejó la silla vacía de Duque marcada con su nombre. Esto ocasionó la reacción de diferentes sectores de gobierno, ciudadanos y políticos. La politóloga Pilar Gaitán añade: "López representa un nuevo liderazgo que convoca y seduce y resuelve problemas (...). Pone la agenda pública y la 'silla vacía' constata el vacío de poder, la precaria gobernabilidad y la pequeñez del Ejecutivo" (Silla Vacía, 2020). Después de este acto, la confrontación pareció menos visible; no obstante, las críticas mutuas entre diferentes entidades de gobierno evidenciaron una brecha de la toma de decisiones.

En los últimos meses, tras la descentralización de las medidas para atender el Covid-19, las tensiones entre los dos niveles de gobierno han tendido a bajar. En este sentido, la toma de decisiones se ha concentrado en el gobierno de Claudia López, aunque continúan los lineamientos nacionales. No obstante, la orientación ideológica de los dos mandatarios amplía las brechas de implementación. En cuanto a las capacidades de Bogotá, se clasifican entre fuerte, débil y no preparada.

Tabla 5

Clasificación de capacidades del gobierno local

\begin{tabular}{|l|l|l|l|}
\hline Dimensión/nivel & $\begin{array}{l}\text { Capacidades } \\
\text { Individuales }\end{array}$ & $\begin{array}{l}\text { Capacidades } \\
\text { Organizacionales }\end{array}$ & Capacidades del Sistema \\
\hline $\begin{array}{l}\text { Competencias } \\
\text { analíticas }\end{array}$ & $\begin{array}{l}\text { 1.Capacidad de } \\
\text { análisis de políticas: } \\
\text { No preparada }\end{array}$ & $\begin{array}{l}\text { 2.Capacidades } \\
\text { organizativas de } \\
\text { información: } \\
\text { Fuerte }\end{array}$ & $\begin{array}{l}\text { 3. Capacidad } \\
\text { del sistema de } \\
\text { conocimientos: } \\
\text { Fuerte }\end{array}$ \\
\hline $\begin{array}{l}\text { Competencias } \\
\text { Operacionales }\end{array}$ & $\begin{array}{l}\text { 4.Capacidad de } \\
\text { conocimientos } \\
\text { especializados: } \\
\text { Débil- Fuerte }\end{array}$ & $\begin{array}{l}\text { 5. Capacidad } \\
\text { de recursos } \\
\text { administrativos: } \\
\text { No preparada a fuerte }\end{array}$ & $\begin{array}{l}\text { 6. Capacidad } \\
\text { del sistema de } \\
\text { responsabilidad y } \\
\text { accountability: } \\
\text { Fuerte }\end{array}$ \\
\hline $\begin{array}{l}\text { Competencias } \\
\text { Políticas }\end{array}$ & $\begin{array}{l}\text { 7. Capacidad de } \\
\text { aumento político: }\end{array}$ & $\begin{array}{l}\text { 8. Capacidad política } \\
\text { organizativa: } \\
\text { Débil }\end{array}$ & $\begin{array}{l}\text { 9. Capacidad del } \\
\text { sistema político- } \\
\text { económico: } \\
\text { Débil }\end{array}$ \\
\hline
\end{tabular}

Fuente: Elaboración propia 


\section{Conclusiones}

La pandemia por Covid-19 dejó en evidencia las (in) capacidades de política de los gobiernos locales para asumir esta crisis con sistemas de salud débiles, bajos presupuestos, y sin ninguna experiencia a diferencia de países asiáticos quienes asumieron la presencia de otros tipos de coronavirus (MERS y SARS) anteriormente. Las capacidades de los gobiernos comenzaron a fortalecerse en la primera etapa de la pandemia, las estrategias de confinamiento se tomaron como un mecanismo para aumentar la capacidad hospitalaria y de atención en salud en el país. Particularmente, Bogotá asumió el aumento en principio de camas UCI y camas hospitalarias para atender a las personas contagiadas, la adecuación del centro de convenciones Corferias, con 2.000 cubículos, aumentó la capacidad de atención; no obstante, se desmanteló antes del primer pico.

Frente a las capacidades organizativas, Bogotá demostró una fuerte interacción entre las instituciones locales que permitieron el vínculo entre ámbitos informáticos en el cruce de información, por ejemplo para la aplicación de subsidios y ayudas a personas en pobreza y pobreza extrema. No obstante, frente al diseño de las medidas en salud en lo referente a las habilidades políticas (astucia y negociación política), la pandemia requirió de respuestas rápidas a un problema de alta complejidad, esto evidenció la necesidad de cooperación entre el sector local y el ámbito del interés nacional.

A pesar del despliegue de las medidas en salud, las cifras continuaron repuntando con casos positivos, para ello se exploró de forma descriptiva las capacidades desplegadas por el gobierno local a partir del modelo de capacidades propuesto por Wu et al, 2015. Frente a las competencias analíticas, Bogotá pasa de un estado (no preparado) debido a la falta de experiencia en la enfermedad a un nivel fuerte, en la comprensión del problema de política en conjunto con expertos. La capacidad organizativa informática logró agregar las bases de datos para cruzar con diferentes instituciones, además de ofrecer datos abiertos sobre la pandemia. Finalmente, la capacidad sistémica del conocimiento se conecta con las anteriores, lo que permite un fuerte conocimiento de la pandemia.

La capacidad de conocimiento especializado, en general en los gobiernos locales, se caracterizó por ser débil a fortalecer las competencias con recursos analíticos, técnicos y operativos, esto vinculado al financiamiento y dotación al servicio de salud. Aunque el sistema de responsabilidad considera lineamientos fuertes de accountability, la condición de la pandemia se asume en un escenario de respuestas rápidas y problemas complejos, que deriva en limitaciones hacia el fortalecimiento de un sistema de responsabilidad.

La capacidad de aumento político comprendió la necesidad de la población con un apoyo político débil con respecto al concejo municipal y a su vez, una capacidad sistémica débil que está asociada al vínculo entre niveles de gobierno. En este sentido, 
las diferencias ideológicas de López y Duque marcaron un derrotero político en los primeros meses de la pandemia, que llevó a fuertes desacuerdos, tensiones y conflictos, trasladados principalmente a las redes sociales. Si bien para resolver las brechas en la toma de decisiones el gobierno nacional centralizó las decisiones, la condición descentralizada en Colombia implicó una toma de decisión conflictiva, como el tema del cierre (o no) del aeropuerto internacional El Dorado, en los primeros días de la pandemia.

Paradójicamente, la coordinación política parece ser sólida entre otros niveles de gobierno, como el regional con la Gobernación de Cundinamarca, otras alcaldías y empresarios. Esto permitió un sumado apoyo a donaciones, financiamiento y fortalecimiento a la red hospitalaria en la ciudad. En cuanto al apoyo ciudadano, la apertura gradual de la economía marcó para el gobierno de Claudia López un corto apoyo del sector comercial, que implicaba acuerdos nacionales y locales frente a la apertura (o no) de establecimientos de consumo.

Para cerrar, este artículo exploratorio propone la apertura de un debate en términos de análisis de política pública, que implica un estudio cualitativo sobre las capacidades de política tanto de gobiernos sub nacionales como centrales al asumir un problema complejo como lo es la pandemia por Covid-19. 


\section{Referencias}

Acosta, L. (2020). Capacidad de respuesta frente a la pandemia de Covid-19 en América Latina y el Caribe. Cepal, 1, 1-15. https://repositorio.cepal.org/bitstream/ handle/11362/45337/4/S2000264_es.pdf

Alcaldía de Bogotá (2020). DECRETO 090 DE 2020. In 19 De Marzo 2020 (Issue 090, p. 16).

Alcaldía de Bogotá, D. (2021). Decreto 135 de 2021. Alcaldía de Bogotá. https://www. alcaldiabogota.gov.co/sisjur/normas/Norma1.jsp?i=109325\&dt=S

Alcaldía de Bogotá, D. (2020). Bogotá tiene capacidad hospitalaria para atender a pacientes críticos de Covid-19. Secretaría de Salud. https://bogota.gov.co/mi-ciudad/salud/bogota-tienecapacidad-hospitalaria-para-atender-pacientes-de-covid-19

Alcaldía de Bogotá, D. (2021). Decreto 148 del 2021. Alcaldía de Bogotá. https://www. alcaldiabogota.gov.co/sisjur/normas/Norma1.jsp?i=109757\&dt=S

Bali, A. S. \& Ramesh, M. (2018). Policy Capacity: A Design Perspective. In M. Howlett \& I. Mukherjee (Eds.), Routledge Handbook of Policy Design (First, pp. 331-344). Routledge.

Cabrero Mendoza, E. (2007). De la descentralización como aspiración a la descentralización como problema. El reto de la coordinación intergubernamental en las políticas sociales. Documentos de Trabajo Del CIDE, 197,37.http://repositorio-digital.cide.edu/bitstream/ handle $/ 11651 / 805 / 80712$.pdf? sequence $=1 \&$ is Allowed $=\mathrm{y}$

Capano, G. (2020). Policy design and state capacity in the COVID-19 emergency in Italy: if you are not prepared for the (un)expected, you can be only what you already are. Policy and Society, 39(3), 326-344. https://doi.org/10.1080/14494035.2020.1783790

Capano, G., Howlett, M., Jarvis, D. S. L., Ramesh, M. \& Goyal, N. (2020). Mobilizing Policy (In)Capacity to Fight COVID-19: Understanding Variations in State Responses. Policy and Society, 39(4), 1-24. https://doi.org/10.1080/14494035.2020.1787628

CEPAL (2020). Medidas y acciones a nivel nacional. Observatorio Covid-19 CEPAL. https:// www.cepal.org/es/temas/covid-19

El Tiempo, R. (2020). Coronavirus: la inoportuna pelea entre Iván Duque y Claudia López. Noticia, 1. https://www.google.com/search?q=Peleas+entre+duque+y+claudia+lopez $\& \mathrm{rlz}=1$ C1EJFC_enCO805EC806\&oq $=$ Peleas + entre + duque $+\mathrm{y}+$ claudia + lopez\&aqs $=$ chrome..69i57j0i22i30.16944j0j7\&sourceid $=$ chrome\&ie $=\mathrm{UTF}-8$

Geert, B., Peters, G. B. \& Koen, V. (2010). The coordination of public sector organizations. Shifting patterns of public management. In Angewandte Chemie International Edition (Vol. 6, Issue 11). Palgrave MacMilan. 
González, E., Hopkins, K., Nagovitch, P., Sonneland, H. \& Zissis, C. (2020). El coronavirus en América Latina. AS/COA, Americas Society Council of the Americas. https://www.ascoa.org/articles/¿̇dónde-está-el-coronavirus-en-américa-latina\#ecuador

Hopkins University, J. (2021). Coronavirus resource center. Gobal Cases. https://coronavirus.jhu. edu/map.html

Howlett, M. (2012). The lessons of failure: Learning and blame avoidance in public policy-making. International Political Science Review, 33(5), 539-555. https://doi. org/10.1177/0192512112453603

Howlett, M. \& Mukherjee, I. (2018). Introduction:The importance of Policy Design: Effective Processes, Tools and Outcomes. In M. Howlett \& M. Ishani (Eds.), Routledge Handbook of Policy Design (First puli, p. 483). Routledge.

Howlett, M. \& Ramesh, M. (2016). Achilles' heels of governance: Critical capacity deficits and their role in governance failures. Regulation and Governance, 10(4), 301-313. https:// doi.org/10.1111/rego.12091

McConnell, A. (2010). Policy success, policy failure and grey areas in-between. Journal of Public Policy, 30(3), 345-362. https://doi.org/10.1017/S0143814X10000152

McConnell, A. (2016). A public policy approach to understanding the nature and causes of foreign policy failure. Journal of European Public Policy, 23(5), 667-684. https://doi.org/ 10.1080/13501763.2015.1127278

Mendieta, D. \& Tobón, M. Luz. (2020). La pequeña dictadura de la COVID-19 en Colombia: uso y abuso de normas ordinarias y excepcionales para enfrentar la pandemia. Opinión Jurídica, 19(40), 243-258. https://doi.org/10.22395/ojum.v19n40a12

Ministerio de Salud, C. (2020a). Circular externa 00005 (p. 9). Ministerio de Salud y Protección social. https://www.minsalud.gov.co/sites/rid/Lists/BibliotecaDigital/RIDENS/PP/PAl/manual-

Ministerio de Salud, C. (2020b). Colombia Confirma su primer caso de Covid- 19. Boletín 050 del 2020. https://www.minsalud.gov.co/Paginas/Colombia-confirma-su-primer-caso-de-COVID-19.aspx

Ministerio de Salud, C. (2020c). Colombia entra a fase de mitigación del Covid-19. Boletín 111 de 2020. https://www.minsalud.gov.co/Paginas/Colombia-entra-en-fase-de-mitigacionde-la-COVID-19.aspx

Ministerio de Salud, C. (2021). Coronavirus Covid-19. Reportes y Tableros de Control. https:// www.minsalud.gov.co/salud/publica/PET/Paginas/Covid-19_copia.aspx

Ministerio del Interior, C. (2020). Decreto 418 de 18 Mar 2020 (p. 3). República de Colombia. https://www.funcionpublica.gov.co/eva/gestornormativo/norma. php?i=110294\#: :text=Dicta medidas transitorias para expedir,19. 
Mukherjee, I. \& Bali, A. S. (2018). Capacities and Customization in Policy Design. In M. Howlett \& I. Mukherjee (Eds.), Routledge Handbook of Policy Design (first, pp. 359-371). Routledge.

Mukherjee, I. \& Bali, A. S. (2019). Policy effectiveness and capacity: two sides of the design coin. Policy Design and Practice, 2(2), 103-114. https://doi.org/10.1080/25741292.201 9.1632616

Newman, J., Cherney, A. \& Head, B. W. (2016). Policy capacity and evidence-based policy in the public service. Public Management Review, 19(2), 157-174. https://doi.org/10.1080 /14719037.2016.1148191

OMS (2020a). Covid-19: Cronología de la actuación de la OMS. Organización Mundial de La Salud. https://www.who.int/es/news/item/27-04-2020-who-timeline---covid-19

OMS (2020b). Report of the WHO-China Joint Mission on Coronavirus Disease 2019 (COVID-19). In The WHO-China Joint Mission on Coronavirus Disease 2019 (Issue February). https://www.who.int/docs/default-source/coronaviruse/who-china-jointmission-on-covid-19-final-report.pdf

OPS, O. P. de S. (2020a). Fortaleciendo Capacidades en el personal de salud al frente de la atención del Covid-19 en Bogotá. Noticias Sobre Covid-19. https://www.paho.org/es/noticias/179-2020-fortaleciendo-capacidades-personal-salud-al-frente-atencion-covid-19-bogota

OPS, O. P. de S. (2020b). Reporte de situación No. 18 (Issue 18, pp. 1-7). OPS.

OPS, O. P. de S. (2021). Informe especial situación Covid-19 en Colombia. Actualización al 24 de enero 2021. In Reporte de Situación No. 205 (Vol. 1, Issue 205).

Painter, M. \& Pierre, J. (2005). Challenges to State Policy Capacity. In M. Painter \& J. Pierre (Eds.), Challenges to State Policy Capacity (1st ed., pp. 1-18). Palgrave MacMilan. https:// doi.org/10.1057/9780230524194

Peters, G. B. (2018). The challenge of policy coordination. Policy Design and Practice, 1(1), 1-11. https://doi.org/10.1080/25741292.2018.1437946

Peters, G. B. \& Pierre, J. (2016). Comparative Governance: Rediscovering the fuctional dimension of governing. In Comparative Governance (1st ed.). Cambridge University Press. https://doi.org/10.1017/cbo9781316681725

Presidencia de la República, de C. (2020). Decreto 417 del 17 de marzo del 2020. In 17 de marzo 2020 (pp. 1-16). https://dapre.presidencia.gov.co/normativa/normativa/ DECRETO 417 DEL 17 DE MARZO DE 2020.pdf

Rodríguez-Cuenca, F., Lemus-Luengas, E. \& Sánchez-Rodríguez, J. (2020). Una mirada al desafio de la pandemia por Coronavirus-Bogotá-2020. 
Saludata, O. de S. de B. (2021). Casos confirmados por Covid-19 en Bogotá. Fichas de Datos. https://saludata.saludcapital.gov.co/osb/index.php/datos-de-salud/enfermedadestrasmisibles/covid19/

Secretaría de Salud, B. (2020). Resolución 712 del 2020. Alcaldía de Bogotá. https://www. alcaldiabogota.gov.co/sisjur/normas/Norma1.jsp?i $=96446 \& \mathrm{dt}=\mathrm{S}$

Secretaría General, A. de B. (2020a). Circular 024 de 2020. Alcaldía de Bogotá. https://www. alcaldiabogota.gov.co/sisjur/normas/Norma1.jsp?i=91306

Secretaría General, A. de B. (2020b). Decreto 081 del 2020. Alcaldía de Bogotá. https://www. alcaldiabogota.gov.co/sisjur/normas/Norma1.jsp?i=91163\&dt=S

Semana, R. (2020). ¿Pelea entre Iván Duque y Claudia López se justifica? Informe, 1. https:// www.semana.com/semana-tv/vicky-en-semana/multimedia/pelea-entre-ivan-duquey-claudia-lopez-en-pandemia-se-justifica-el-debate/686635/

Silla Vacía, web. (2020). Con la silla vacía López opaca su gesto de reconciliación. Editorial. https:// lasillavacia.com/silla-vacia-lopez-opaca-su-gesto-reconciliacion-78408

WHO (2020). Transmission of SARS-CoV-2: implications for infection prevention precautions. Who, 1(March), 1-10. https://www.who.int/news-room/commentaries/ detail/transmission-of-sars-cov-2-implications-for-infection-preventionprecautions\%0Ahttps://bityli.com/j84ms

Woo, J.J. (2020). Policy capacity and Singapore's response to the COVID-19 pandemic. Policy and Society, 39(3), 345-362. https://doi.org/10.1080/14494035.2020.1783789

Wu, X., Ramesh, M. \& Howlett, M. (2015). Policy capacity: A conceptual framework for understanding policy competences and capabilities. Policy and Society, 34(3-4), 165-171. https://doi.org/10.1016/j.polsoc.2015.09.001

Wu, X., Ramesh, M. \& Howlett, M. (2018a). Policy Capacity: Conceptual Framework and Essential Components. In X. Wu, M. Howlett \& M. Ramesh (Eds.), Policy Capacity and Governance (pp. 1-25). Palgrave MacMilan. https://doi.org/10.1007/978-3-31954675-9_1

Wu, X., Ramesh, M. \& Howlett, M. (2018b). Policy Capacity and Governance. Policy Capacity and Governance, 1-25. https://doi.org/10.1007/978-3-319-54675-9

Enviado: 04 de mayo de 2021

Aceptado: 02 de julio de 2021 
